Check for updates

Cite this: Phys. Chem. Chem. Phys., $2018,20,14708$

Received 6th December 2017 Accepted 2nd April 2018

DOI: $10.1039 / c 7 c p 08195 b$

rsc.li/pccp

\section{Sequential and direct ionic excitation in the strong-field ionization of 1-butene molecules}

\author{
Felix Schell, ${ }^{a}$ Andrey E. Boguslavskiy, ${ }^{\text {bc }}$ Claus Peter Schulz, ${ }^{a}$ Serguei Patchkovskii, ${ }^{a}$ \\ Marc J. J. Vrakking, ${ }^{a}$ Albert Stolow ${ }^{b c d}$ and Jochen Mikosch (D) *ab
}

\begin{abstract}
We study the Strong-Field lonization (SFI) of the hydrocarbon 1-butene as a function of wavelength using photoion-photoelectron covariance and coincidence spectroscopy. We observe a striking transition in the fragment-associated photoelectron spectra: from a single Above Threshold Ionization (ATI) progression for photon energies less than the cation $D_{0}-D_{1}$ gap to two ATI progressions for a photon energy greater than this gap. For the first case, electronically excited cations are created by SFI populating the ground cationic state $D_{0}$, followed by sequential post-ionization excitation. For the second case, direct sub-cycle SFI to the $D_{1}$ excited cation state contributes significantly. Our experiments access ionization dynamics in a regime where strong-field and resonance-enhanced processes can interplay.
\end{abstract}

\section{Introduction}

Attosecond science derives from highly non-linear interactions of light and matter, initiated near the electric field maxima of intense femtosecond laser pulses. ${ }^{1}$ Strong-Field Ionization (SFI) underpins all of attosecond science. ${ }^{2}$ It initiates the three-step process of High Harmonic Generation (HHG), leading to the production of attosecond pulses in the extreme ultraviolet (XUV) spectral region. ${ }^{3,4}$ It also underlies most strong-field spectroscopies of ultrafast dynamics in atoms and molecules. ${ }^{5-9}$ SFI is a sub-cycle process, wherein continuum electron wave packets produced in every other half-cycle of a multi-cycle laser pulse interfere to form a comb-like Above Threshold Ionization (ATI) photoelectron spectrum. ${ }^{10,11}$

As attosecond science moves into the realm of polyatomic molecules, the many-electron and non-adiabatic nature of strongfield spectroscopies becomes important. ${ }^{12-18}$ An example is SFI directly to different electronic states of the cation, which - in the Koopmans' picture - is interpreted as simultaneous (i.e. same field cycle) ionization from the highest occupied molecular orbital (HOMO) as well as from lower-lying orbitals (HOMO-1, HOMO-2, etc.). ${ }^{13,19-23}$ Initially controversial, this phenomenon is now becoming widely accepted. Importantly, direct (i.e. sub-cycle) SFI to different electronic states can initiate attosecond electronic dynamics in atoms and molecules. ${ }^{24-26}$

\footnotetext{
${ }^{a}$ Max-Born-Institut, Max-Born-Strasse 2A, 12489 Berlin, Germany. E-mail: jochen.mikosch@mbi-berlin.de

${ }^{b}$ National Research Council, 100 Sussex Drive, Ottawa, ON, K1A OR6, Canada ${ }^{c}$ Univ. Ottawa, Dept. Phys., 150 Louis Pasteur, Ottawa, ON, K1N 6N5, Canada

${ }^{d}$ Univ. Ottawa, Dept. Chem., 10 Marie Curie, Ottawa, ON, K1N 6N5, Canada
}

Direct observation of SFI populating excited cation states is achieved via Channel-Resolved Above Threshold Ionization (CRATI). ${ }^{13}$ In its most basic implementation, CRATI relies on the fact that, in many polyatomic molecules, only the ground $\left(D_{0}\right)$ state of the cation is stable with respect to spontaneous (i.e. field-free) unimolecular fragmentation. The excitation of higher-lying electronic states of the cation inevitably leads to dissociation. However, while the appearance of ionic fragments in the mass spectrum is a sign of the population of excited cation states, there are, in general, three dissociative SFI pathways that need to be distinguished: (i) direct SFI to excited cation states; (ii) SFI to the ground cationic state followed by post-ionization excitation; (iii) strong-field excitation of the neutral molecule followed by ionization to cation excited states. In both cases (ii) and (iii), the laser excitation includes sequential events, which may be separated in time by one or more cycles of the laser field. The CRATI method disentangles direct (i) from sequential (ii, iii) channels by measuring Photoelectron kinetic Energy Spectra (PES) correlated to different ions in the mass spectrum (parent or fragment ions) using coincidence or covariance techniques. $^{27-29}$ The PES from SFI is comprised of an ATI comb, i.e. a series of equidistant peaks separated by the photon energy and related to the above-threshold (i.e. above ionization potential (IP)) absorption of integer numbers $n$ of photons of energy $h \nu$ :

$$
E_{\text {kin }}=n \times h \nu-\mathrm{IP}-U_{\mathrm{p}} .
$$

The ATI comb is phase-shifted in photoelectron energy by two terms: the channel-specific IP, which may include dynamic Stark shifts; the intensity-dependent ponderomotive potential $\left(U_{\mathrm{p}}\right)$, which describes the effective pseudopotential experienced by the freed electron in the oscillating laser field. Using CRATI, we previously demonstrated that the formation of several ionic 
fragments in 1,3-butadiene and $n$-butane is associated with direct sub-cycle SFI populating different cation electronic states (pathway (i)). ${ }^{13}$ This was confirmed by the fact that ATI combs correlated with fragment ions (indicative of the formation of an excited cation electronic state) were shifted with respect to the ATI comb correlated with the ground cation state, with the shift being equal to the difference in vertical IP between these channels.

Recently, the low energy region of the SFI PES (up to about $2 \mathrm{eV}$ energy) was investigated in a series of publications using coincidence velocity map imaging (VMI). ${ }^{30-34}$ Direct and sequential population pathways to dissociative cation states were discerned for a range of molecular systems ${ }^{31}$ at a fixed SFI wavelength of $800 \mathrm{~nm}$. The molecules were characterized by their gap energy, defined as the energy difference between the energetically lowest-lying dissociative cation electronic state and the energetically highest-lying stable cation electronic state. The observation was made that direct SFI to electronically excited dissociative states strongly decreases as this energy gap increases. This finding was rationalized within the framework of the strong field approximation (SFA), according to which the ionization rate is proportional to the product of the Dyson norm (in the simplest semi-classical approximation) and the Keldysh ionization rate. A Dyson orbital represents the one-electron wave function obtained by projecting an $N-1$ electron wave function of the ion onto the $N$ electron wave function of the neutral molecule. A large norm of a Dyson orbital implies that the electronic states of the ion and neutral have a similar electronic configuration. Hence, if it is assumed that the Dyson norms are molecule-independent, the channel-dependent ionization rates differ by the ratio of the Keldysh ionization rates. ${ }^{35}$ Keeping only the leading order in the IP difference $\Delta \mathrm{IP}$ leads to a ratio of $\kappa=\exp \left[-2\left(2 \mathrm{IP}_{0}\right)^{1 / 2} \Delta \mathrm{IP} / F\right]$, where $\mathrm{IP}_{0}$ is the lower of two channelspecific IPs and $F$ is the field strength. However, the authors also point out that the experiments indicate a threshold behaviour. Direct SFI to the excited cation state was found to be comparable in magnitude to SFI producing the ionic ground state for gap energies below the photon energy, whereas the direct excited state SFI channel is generally rather weak for gap energies above the photon energy. ${ }^{31}$ Such a threshold behaviour is not captured by the simple SFA model.

Variation of the target molecule as used in ref. 31, however, introduces many critical changes in molecular properties, including variations in the point group symmetry, electronic structure, density of states, transition dipoles, polarizabilities and electronic level spacings. Importantly, these variations may each affect the SFI rates and channel-resolved yields. Motivated by the work in ref. 31, here we present an SFI study of a single molecule, the hydrocarbon 1-butene $\left(\mathrm{C}_{4} \mathrm{H}_{8}\right)$, but now systematically tune the photon energy, in three steps, from below to above the gap energy of $2.0 \mathrm{eV} .^{36,37}$ This approach avoids the complicated effects of variation of important molecular properties in the experiment.

1-Butene has two conformers which co-exist at room temperature: skew (gauge) $(\approx 70 \%$ abundance) and syn (cis) $(\approx 30 \%$ abundance). ${ }^{36}$ For supersonic expansion conditions, only the skew conformer is present, ${ }^{38}$ owing to the small enthalpy difference of only about $0.24 \mathrm{kcal} \mathrm{mol}^{-1}(10 \mathrm{meV}) .{ }^{39}$ Regardless, there is no significant difference in the field-free IPs for ionization to the cation ground state and the lowest excited cation states. ${ }^{36}$

\section{Experimental}

Measurements at wavelengths of 795 and $634 \mathrm{~nm}$ were performed in a magnetic bottle Photoelectron Photoion COincidence (PEPICO) instrument (NRC, Ottawa), described previously. ${ }^{40}$ In brief, the 1-butene target molecules were provided by a doubly skimmed, $1 \mathrm{~mm}$ diameter continuous molecular beam of seeded helium ( $1 \%$ admixture). The molecular beam was intersected at $90^{\circ}$ by a femtosecond laser beam focused with a $500 \mathrm{~mm}$ focal length concave mirror. Photoelectrons and positively charged photoions were extracted from the interaction region into opposing collinear flight tubes. For each photoelectron, its kinetic energy was measured in a magnetic bottle spectrometer, whereas for each ion, its mass was measured in a time-of-flight (TOF) spectrometer. Electrons and ions were detected by triple-stack micro-channel plates (MCPs), yielding narrow pulse height distributions. The overall probability of detection was $\approx 50 \%$ for both photoelectrons and -ions. Experiments were performed at a typical SFI event rate of between one and two per laser shot. A covariance analysis was performed as described in ref. 27 and 28 , yielding PES correlated with each ion in the mass spectrum.

Experiments at $\lambda=795 \mathrm{~nm}$ utilized a laser beam directly from a Titanium:Sapphire (Ti:Sa) amplified femtosecond laser system (Legend Elite Duo, Coherent). The pulse duration of $35 \mathrm{fs}$ was determined with a home-built single-shot autocorrelator. ${ }^{41}$ For experiments at $\lambda=634 \mathrm{~nm}$, the fundamental beam from the laser system was converted in an Optical Parametric Amplifier (TOPAS-HE, Light Conversion), whose signal beam was frequency-doubled in a $150 \mu \mathrm{m}$ thick BBO crystal to obtain the SFI laser pulse. Based on the pulse duration of the signal beam, measured by cross-correlation with the fundamental laser pulse, we estimated a pulse duration of $35 \mathrm{fs}$ for the $634 \mathrm{~nm}$ pulse in the interaction region with the molecular beam. The intensities reported for $\lambda=795$ and $634 \mathrm{~nm}$ (see Table 1) were determined by an independent measurement of the ponderomotive energy $U_{\mathrm{p}}$, which is proportional to the intensity. To this end, the absolute position of the ATI comb originating from $\mathrm{D}_{0}$ SFI of background $\mathrm{H}_{2} \mathrm{O}$ molecules was determined. These were present in the 1-butene SFI mass spectra at very small abundance. For the intensity range employed, the Stark shift of the $\mathrm{D}_{0}$ IP of water

Table 1 Wavelengths $\lambda$, photon energies $E_{h \nu}$ intensities 1 , laser pulse durations $\tau$, and Keldysh parameters $\gamma$ (calculated using the $D_{0}$ and the $D_{1}$ $I P$, respectively) employed in the present study of SFI of 1-butene. In the last column, the experimentally measured contribution of the parent ion to the total 1-butene-related ion yield $f_{\mathrm{p}}$ is given. The Keldysh parameters are based on the known experimental ground-state, field-free IP $=9.77 \pm 0.01 \mathrm{eV}^{42}$ and the experimentally known $2.0 \mathrm{eV}$ energy difference between $D_{0}$ and $D_{1}{ }^{36,37}$ The uncertainty of the reported intensities is estimated to be about $30 \%$

\begin{tabular}{llllll}
\hline$\lambda\left(E_{h \nu}[\mathrm{eV}]\right)$ & $I\left[\mathrm{~W} \mathrm{~cm}{ }^{-2}\right]$ & $\tau[\mathrm{fs}]$ & $\gamma_{\mathrm{D}_{0}}$ & $\gamma_{\mathrm{D}_{1}}$ & $f_{\mathrm{p}}$ \\
\hline $795 \mathrm{~nm}(1.56)$ & $1.5 \times 10^{13}$ & 35 & 2.3 & 2.5 & 0.7 \\
$634 \mathrm{~nm}(1.95)$ & $2.5 \times 10^{13}$ & 35 & 2.3 & 2.5 & 0.4 \\
$401 \mathrm{~nm}(3.09)$ & $\approx 1 \times 10^{13}$ & 30 & & $\approx 6$ &
\end{tabular}


is known to be negligible. ${ }^{13}$ While from the ATI comb position the ponderomotive shift can only be derived modulo the photon energy (see eqn (1)), the intensity calibration becomes unique by analyzing measurements as a function of pulse energy. Due to the nature of the calibration, the intensities reported in this work are actual intensities, averaged over the focal volume region where charged particles were produced by SFI.

Experiments at a wavelength of $401 \mathrm{~nm}$ were performed in a REaction MIcroscope (REMI) instrument ${ }^{43}$ (MBI, Berlin). The output of a Ti:Sa amplified femtosecond laser system operating at $10 \mathrm{kHz}$ repetition rate (Aurora, Amplitude Technologies) was frequency-doubled in a $200 \mu \mathrm{m}$ thick BBO crystal. After separating the fundamental with a pair of dichroic beamsplitters, a concave aluminium-coated mirror with a focal length of $500 \mathrm{~mm}$ was used to focus the $401 \mathrm{~nm}$ beam into the interaction region of the REMI. These relatively loose focusing conditions matched the ones used in the PEPICO experiment and were implemented to minimize longitudinal intensity averaging. The employed laser focus features a Rayleigh range greater than the tightly skimmed molecular beam diameter. In the REMI, the focused laser beam intersected a doubly differentially pumped continuous molecular beam of 4\% 1-butene in helium. The beam originated from a $10 \mu \mathrm{m}$ diameter nozzle, using backing pressures in the range of 0.4-1.5 bar. Parallel homogeneous electric $\left(590 \mathrm{~V} \mathrm{~m}^{-1}\right)$ and magnetic (4.2 Gauss) fields guided the charged particles created in the interaction region to a pair of opposing position-sensitive MCP detectors (Roentdek). From the TOF of the particles and their impact position on the detector, along with the known field strengths, the initial momentum vector - and thus the kinetic energy - can be reconstructed for each particle using Newtonian mechanics. For ions, the TOF additionally yields information on the mass of the detected ion (see below). In order to be able to assign the particles detected after a laser shot to the same ionization event, it is necessary to ensure that at most one molecule is ionized by each shot. For this reason, the rate of ionization was limited to at most 0.2 events per shot, allowing the determination of PES in coincidence with different ionic species. The measured spectrum of the $401 \mathrm{~nm}$ pulse ( $8 \mathrm{~nm}$ FWHM) supported a transform-limited pulse duration of 30 fs. The spectral phase of the fundamental was optimized with a commercial pulse shaping system (Dazzler, Fastlite) in the laser by maximizing the count rate from SFI in the REMI. Therefore, the minimum $401 \mathrm{~nm}$ pulse duration in the interaction region is expected to be close to the transform limit. The SFI intensity was estimated using a measurement of the focal spot size in the interaction region performed with a beam profile camera (Ophir Spiricon). The result is consistent with the ponderomotive shift of the ATI comb that we observed for the 1,3-butadiene parent ion, which was present at small quantity as an impurity in the mass spectrum (see below). For a measurement with a temporally stretched pulse, $12 \mathrm{~mm}$ of UV fused silica glass were inserted into the $401 \mathrm{~nm}$ beam path before the focusing mirror without adjustment of the pulse shaper. The glass stretches a transform-limited 30 fs duration $401 \mathrm{~nm}$ pulse to a duration of $110 \mathrm{fs}$ by chromatic dispersion, ${ }^{44}$ while an estimation of the B-integral shows that non-linear phase-shifts are expected to be negligible. The pulse energy was adjusted to maintain the parent ion ATI comb position and thus keep the intensity the same for both measurements.

Ion TOF spectra obtained with both experimental setups contain information on the mass of the detected ions and their kinetic energy. The PEPICO instrument is designed for mass separation and features a much higher electrostatic extraction field. Well-isolated and identifiable mass peaks are clearly visible for all fragments in the PEPICO TOF spectrum. In contrast, the REMI instrument is designed for momentum resolution and features a weak electrostatic extraction field. Sharp peaks in the TOF spectrum occur only for parent ions. Kinetic energy release from the dissociation of the molecule leads to strongly broadened mass peaks for the fragments in the REMI TOF spectra. Clearly distinguishable, however, are fragment groups $\mathrm{C}_{x} \mathrm{H}_{n}{ }^{+}$, containing the same number $x$ of $\mathrm{C}$ atoms but different numbers $n$ of $\mathrm{H}$ atoms. For the REMI data presented in this work (401 nm wavelength), PES coincident with such fragment groups containing a fixed number of $\mathrm{C}$ atoms are shown.

Mass separation of ions via TOF following acceleration in an electric field is sensitive to the mass-to-charge ratio. We have analyzed the fragment-resolved electron-to-ion ratio and obtained values close to unity for the conditions in this work. We hence conclude that there is no significant contribution from double ionization, as is to be expected for the moderate intensities employed here. We also note that the intensities employed in this work were far below the saturation intensities and that only a very small fraction of the $\approx 10^{3}$ 1-butene molecules present in the laser focus were ionized.

Importantly, the CRATI method must avoid a strong contribution of false covariances which can correlate all photoelectron spectra with a source of noise in the experiment. Such false covariances could artificially 'contaminate' the fragment ATI spectra with 'replicas' of the parent ATI spectrum. ${ }^{28} \mathrm{We}$ explicitly ruled out such effects in our data by analyzing the PES correlated with $\mathrm{C}_{4} \mathrm{H}_{6}{ }^{+}$in the mass spectrum, which can be traced to a small impurity of 1,3-butadiene in the 1-butene gas sample. A small quantity of 1,3-butadiene is both specified by the gas supplier as well as confirmed by a Nuclear Magnetic Resonance (NMR) trace gas analysis. In our data, we find that the ATI spectrum associated with $\mathrm{C}_{4} \mathrm{H}_{6}{ }^{+}$is clearly shifted with respect to the ATI spectra originating from SFI of 1-butene. The shift corresponds to the difference in ground-state IP between 1,3-butadiene and 1-butene and confirms that our data are not contaminated by false covariances.

\section{Results}

We studied SFI of 1-butene at $\lambda=795 \mathrm{~nm}\left(E_{h \nu}=1.56 \mathrm{eV}\right)$ and $\lambda=634 \mathrm{~nm}\left(E_{h \nu}=1.95 \mathrm{eV}\right)$ for intensities of $I=1.5 \times 10^{13} \mathrm{~W} \mathrm{~cm}^{-2}$ and $I=2.5 \times 10^{13} \mathrm{~W} \mathrm{~cm} \mathrm{~cm}^{-2}$, respectively $(30 \%$ estimated uncertainty). The laser pulse duration was $\tau \approx 35$ fs. The resulting Keldysh parameter $\gamma=\sqrt{\mathrm{IP} /\left(2 U_{\mathrm{p}}\right)^{35}}$ for $\mathrm{D}_{0}$ SFI was $\gamma_{D_{0}}=2.3$ for both cases (see Table 1 ). The $\mathrm{C}_{4} \mathrm{H}_{8}{ }^{+}$parent is found to be the dominant ion in the mass spectrum under the 
(a) $795 \mathrm{~nm}$

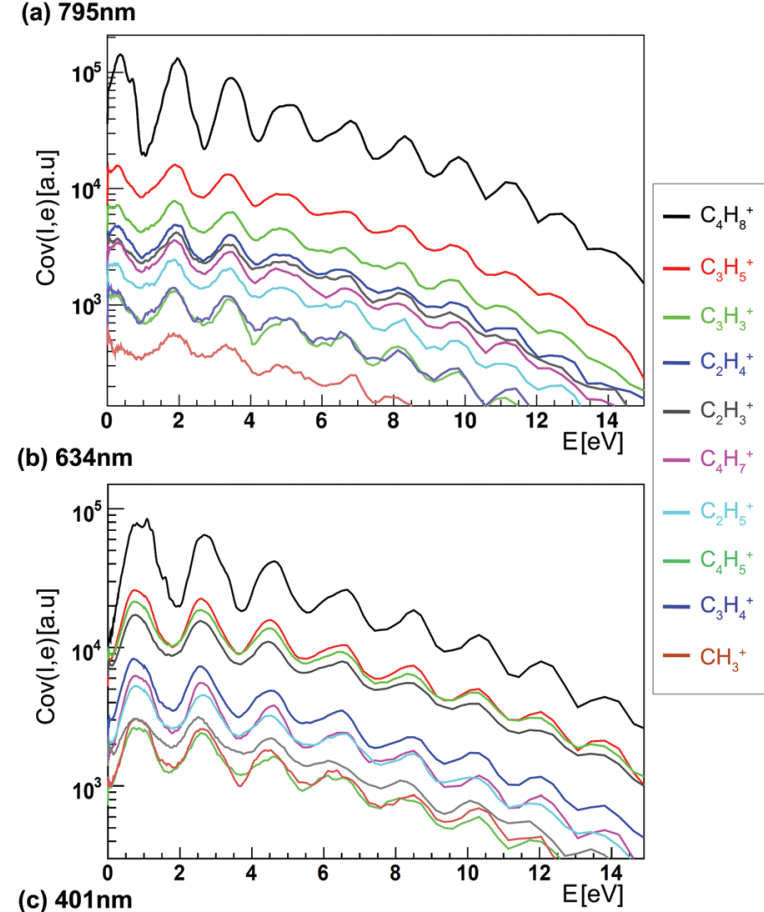

(c) $401 \mathrm{~nm}$

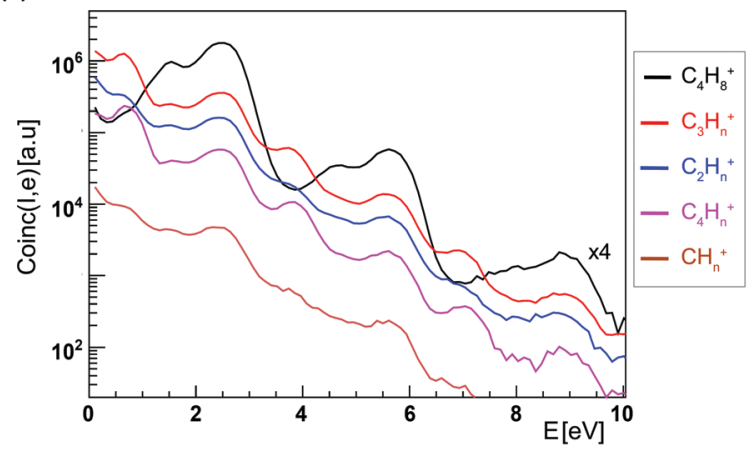

Fig. 1 Correlated PES for the parent ion (black) and selected fragment ions for SFI of 1-butene at $\lambda=795 \mathrm{~nm}$ (a), $634 \mathrm{~nm}$ (b), and $401 \mathrm{~nm}$ (c). The color coding is the same in panels (a) and (b), which feature a shared legend. The laser parameters used in this study are summarized in Table 1.

employed conditions. In Fig. 1(a) (795 nm) and (b) (634 nm), we show the covariant PES for the parent ion (black) and for some of the most prominent fragment ions. Each spectrum features a comb of peaks spaced by the photon energy, as expected for ATI. Importantly, in both the 795 and the $634 \mathrm{~nm}$ measurement we observe that the ATI peak positions in all fragment spectra line up (i.e. same comb shift) with those of the parent ion. Other fragments (not shown) display a similar PES, except for mass 54, which is traced back to a 1,3-butadiene impurity (see above). The integral over the covariant PES corresponds to the abundance of the respective ion in the ion TOF spectrum multiplied by the detection efficiency-corrected electron-to-ion ratio for that particular ion. The electron-to-ion ratios are unity to within $5 \%$ for all fragments in Fig. 1(a), except for $\mathrm{CH}_{3}$ (which shows an elevated electron/ion ratio of up to about 1.3), confirming that all fragments, except potentially the very lightest, are correlated with single ionization.
Furthermore, using the REMI, we studied SFI of 1-butene at $\lambda=401 \mathrm{~nm}\left(E_{h \nu}=3.1 \mathrm{eV}\right)$, at a comparable pulse duration of $30 \mathrm{fs}$ and an intensity of $\approx 1 \times 10^{13} \mathrm{~W} \mathrm{~cm}^{-2}(\gamma \approx 6$, see Table 1$)$. In Fig. 1(c), we plot the PES coincident with the parent ion and with groups of fragments $\mathrm{C}_{x} \mathrm{H}_{n}{ }^{+}$, which differ by the number $x$ of carbon atoms. As can be seen, the PES coincident with the parent ion (black) contains a progression of wide peaks, spaced by the photon energy. The wide peaks feature two maxima, separated by about $0.9 \mathrm{eV}$. The weaker maximum is located on the low kinetic energy side of the wide peak. The fragment PES exhibit an ATI progression which lines up with the dominant maximum in the parent PES. In the lowest order of the comb, small peaks are also visible at the location of the weaker maximum in the parent PES (see structures at $1.4 \mathrm{eV}$ photoelectron kinetic energy). Importantly, in contrast to the cases of $\lambda=795 \mathrm{~nm}$ and $\lambda=634 \mathrm{~nm}$, a second ATI progression is clearly visible in the fragment-correlated PES, which is absent in the parent-correlated PES, with maxima that appear around the minima in the parent ion ATI comb. This second comb is particularly pronounced for PES coincident with the $\mathrm{C}_{3} \mathrm{H}_{n}{ }^{+}$(red) and the $\mathrm{C}_{4} \mathrm{H}_{n}{ }^{+}$(magneta) fragment groups. We also note that overall the exponential decrease of the yield with ATI order is much stronger in the $401 \mathrm{~nm}$ data, as compared to the 795 and $634 \mathrm{~nm}$ data, consistent with the lower intensity employed at $\lambda=401 \mathrm{~nm}$.

For further clarification and analysis, we removed the exponential dependence of the signal intensity on the ATI order. ${ }^{45}$ Typically, two different exponentials were used to describe the non-oscillatory background of the PES for the lower and for the higher photoelectron energies in Fig. 1, which was subtracted from the signal. In Fig. 2, we plot the resulting spectra for the parent ion (upper panels) and the most abundant fragment $\mathrm{C}_{3} \mathrm{H}_{5}{ }^{+} / \mathrm{C}_{3} \mathrm{H}_{n}{ }^{+}$(lower panels) for $\lambda=795 \mathrm{~nm}(\mathrm{a}), 634 \mathrm{~nm}(\mathrm{~b})$ and $401 \mathrm{~nm}$ (c), respectively. The small deviations of the peak separations from the photon energy, evident from the dashed vertical lines that are spaced by the photon energy, are attributed to slight imperfections in the (independent) energy calibrations. The additional comb in the $\mathrm{C}_{3} \mathrm{H}_{n}{ }^{+}$fragment ATI spectrum, marked in red, is obvious in the $401 \mathrm{~nm}$ data. This secondary ATI progression is downshifted by $1.8 \mathrm{eV}$ relative to the dominant ATI comb.

We studied the dependence of the secondary ATI comb on the $401 \mathrm{~nm}$ pulse duration $\tau$ by temporally stretching (i.e. linearly chirping) the pulse from $\tau=30$ fs to $\tau=110 \mathrm{fs}$, concomitantly adjusting the pulse energy so as to maintain constant intensity. In Fig. 3, we show the PES coincident with $\mathrm{C}_{3} \mathrm{H}_{n}{ }^{+}$fragments for SFI with a $30 \mathrm{fs}$ pulse (red) and a $110 \mathrm{fs}$ pulse (black). In both curves, the lowest order of the additional comb structure that is only visible in the fragment-correlated PES and which is assigned to $\mathrm{D}_{1}$ ATI peaks (see below) is normalized to the same value. The relative contribution of the dominant $\mathrm{D}_{0}$ ATI comb (see below) is significantly higher for the increased pulse duration.

Finally, for $\lambda=795 \mathrm{~nm}$, we investigated the intensity dependence of the ATI spectrum correlated with the parent ion and the $\mathrm{C}_{3} \mathrm{H}_{5}{ }^{+}$fragment, shown in Fig. 4(a) and (c). When the 
(a) 795nm

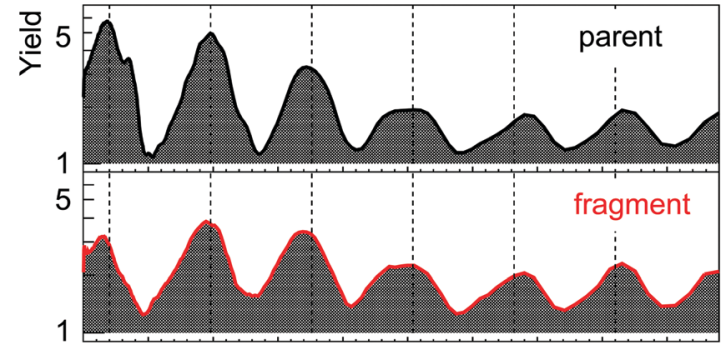

(b) $634 \mathrm{~nm}$

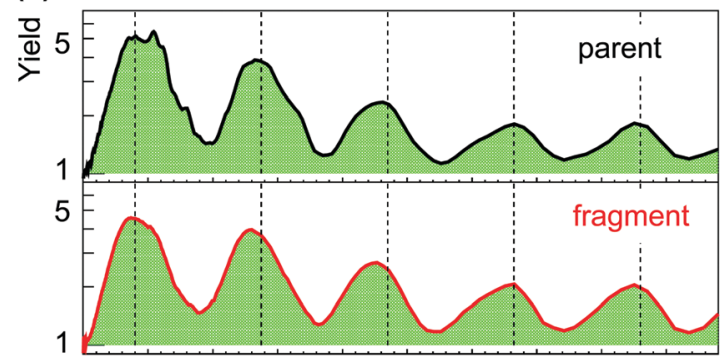

(c) $401 \mathrm{~nm}$

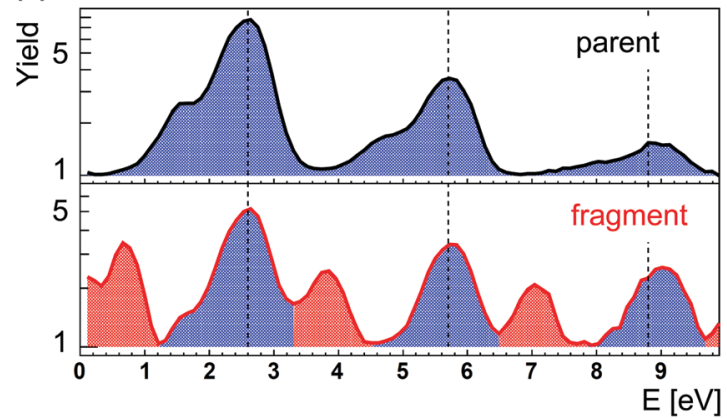

Fig. 2 Oscillatory part of the PES from Fig. 1 (obtained by subtraction of the background, see text) correlated to the parent ion (upper panels) and the dominant $\mathrm{C}_{3} \mathrm{H}_{5}{ }^{+} / \mathrm{C}_{3} \mathrm{H}_{n}{ }^{+}$fragment ion (lower panels), for the three employed SFI wavelengths. The spectra are derived from Fig. 1 and are normalized to the same area. The vertical dashed lines are spaced by the photon energy.

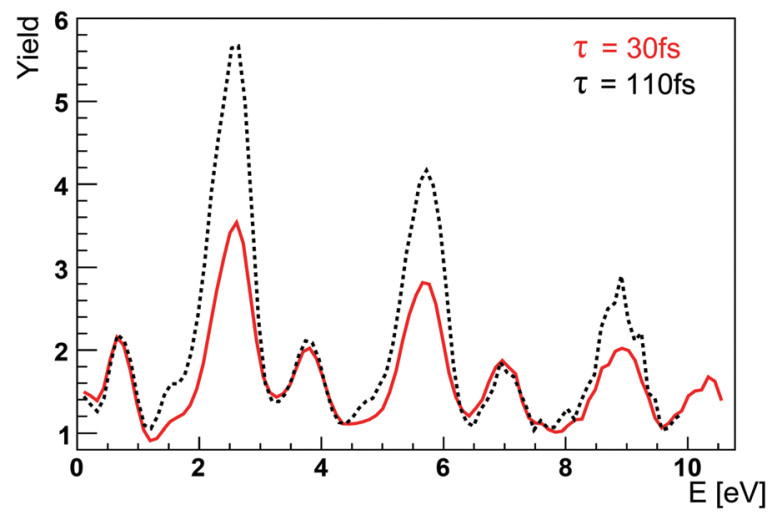

Fig. 3 Oscillatory part of the PES (obtained by subtraction of the background, see text) correlated to the $\mathrm{C}_{3} \mathrm{H}_{n}{ }^{+}$fragment ion group for $\mathrm{SFI}$ at $\lambda=401 \mathrm{~nm}$, with laser pulse durations of $30 \mathrm{fs}$ (solid red) and $110 \mathrm{fs}$ (dashed black) at a constant peak intensity. An overall scaling factor was applied to the spectra such that the lowest-order $D_{1}$ ATI peaks at $E \approx 0.7 \mathrm{eV}$ are equal in amplitude.
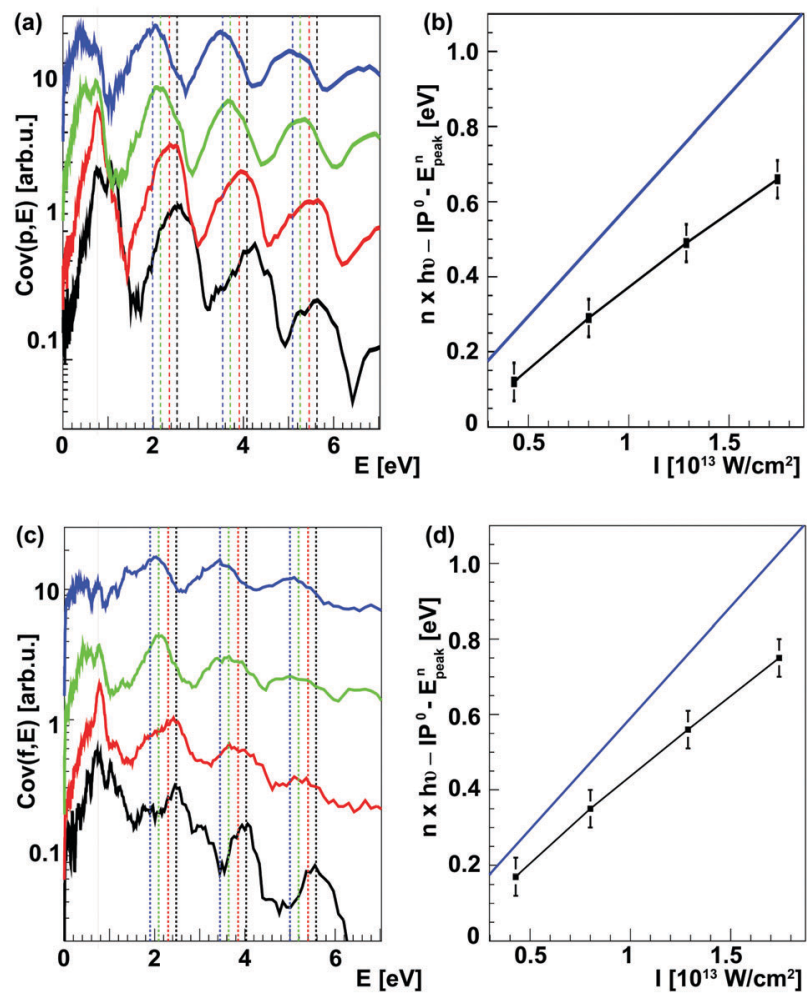

Fig. 4 Covariant parent (a) and $\mathrm{C}_{3} \mathrm{H}_{5}{ }^{+}$fragment (c) ATI spectra $(\lambda=795 \mathrm{~nm})$ at a fixed pulse duration of $\tau=108$ fs for intensities of 0.43 (black), 0.80 (red), 1.3 (green), and $1.7 \times 10^{13} \mathrm{~W} \mathrm{~cm}^{-2}$ (blue). The dashed vertical lines mark the centres of the ATI peaks for each photon order $n$, colored so as to correspond to their associated laser intensity. ( $b$ and $d$ ) Data points representing the absorbed photon energy minus the field-free vertical ionization potential I $P^{0}$ ( $D_{0}$ ionization) and the measured photoelectron kinetic energy for the marked peak positions of panel (a) and (c). The calculated ponderomotive energy as a function of intensity is shown as the solid blue line. Whereas the error on the relative intensity of the different data points is negligible, there is a systematic uncertainty of $30 \%$ for the entire set of measurements, resulting from the absolute intensity calibration.

intensity was systematically increased from $0.4 \times 10^{13} \mathrm{~W} \mathrm{~cm}^{-2}$ (black) to $1.7 \times 10^{13} \mathrm{~W} \mathrm{~cm}^{-2}$ (blue), the ATI comb shifted to lower photoelectron kinetic energies. This shift is quantitatively compared to the ponderomotive shift, as discussed below.

\section{Discussion}

In 1-butene, only the electronic ground state of the cation is stable: All excited cationic states undergo unimolecular fragmentation. ${ }^{46}$ A preponderance of the parent ion $\mathrm{C}_{4} \mathrm{H}_{8}{ }^{+}$in the mass spectrum therefore implies that SFI populates mostly the $\mathrm{D}_{0}$ continuum. The relative contribution of the parent ion to the total 1-butene-related ion yield, $f_{\mathrm{p}}$, sets a lower limit to the relative $\mathrm{D}_{0}$ SFI yield. In turn, the appearance of fragments is a signature of the formation of electronically excited $\mathrm{C}_{4} \mathrm{H}_{8}{ }^{+}$. Whether the ground and the electronically excited cation states are populated by direct SFI from the neutral ground state, as in the case of 1,3-butadiene $\left(\mathrm{C}_{4} \mathrm{H}_{6}{ }^{+}\right),{ }^{13}$ or via a sequential process of ionization and excitation (starting with ionization or excitation), cannot be determined from the mass 
(a) $795 \mathrm{~nm}$

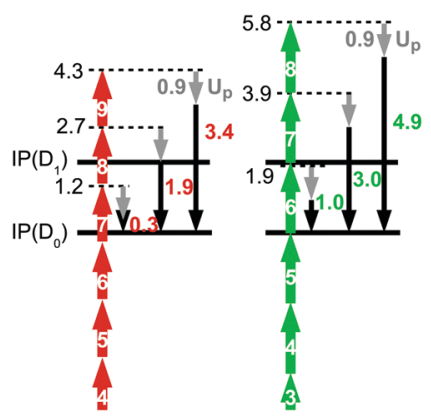

(c1) $401 \mathrm{~nm}$

(c2) $401 \mathrm{~nm}$

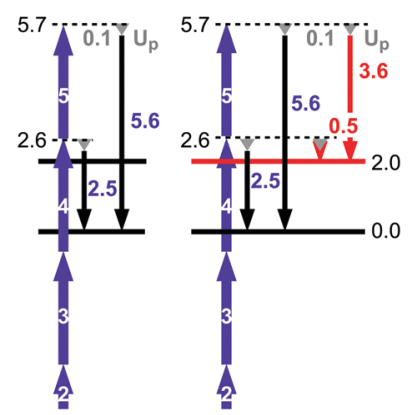

Fig. 5 Wavelength-dependent SFI schemes for ATI of 1-butene molecules. For a discussion, see the text.

spectrum alone. As seen from Table 1 , we find that the measured $f_{\mathrm{p}}$ decreases from 0.7 for $\lambda=795 \mathrm{~nm}$, to 0.4 for $\lambda=634 \mathrm{~nm}$, and 0.2 for $\lambda=401 \mathrm{~nm}$. We can conclude from these numbers that the sum of direct and indirect population of electronically excited cation states increases strongly for decreasing wavelength, despite the lower intensity employed at $\lambda=401 \mathrm{~nm}$.

Given that the $\mathrm{C}_{4} \mathrm{H}_{8}{ }^{+}$parent ion singles out the stable ionic ground state of 1-butene, the PES correlated with the parent ion represents the $\mathrm{D}_{0}$ SFI ATI spectrum (Fig. 2 upper panels). It is instructive to quantitatively consider the expected comb positions from eqn (1), as schematically depicted in Fig. 5. In this analysis, we include the known experimental value of $\operatorname{IP}^{0}\left(D_{0}\right)=9.77 \pm 0.01 \mathrm{eV}$ (vertical, field-free), ${ }^{42}$ the experimentally known $2.0 \mathrm{eV}$ energy difference between the vertical $\mathrm{D}_{0}$ and $\mathrm{D}_{1}$ ionization energies, ${ }^{36,37}$ the respective photon energies (lengths of colored arrows) and the independently determined ponderomotive potential $U_{\mathrm{p}}$ shift (grey arrows). The expected ATI peak positions for direct $\mathrm{D}_{0}$ SFI (length of black arrows) are denoted in units of $\mathrm{eV}$, for the case of $\lambda=795 \mathrm{~nm}$ (panel (a), red) and $\lambda=634 \mathrm{~nm}$ (panel (b), green), respectively. As seen by comparing with Fig. 2(a) and (b), the calculated positions of the ATI combs agree well with the measurement for these photon energies. The observed deviations of about $0.2 \mathrm{eV}$ are consistent with the fact that the differential Stark shift for $\mathrm{D}_{0}$ SFI (i.e. the difference between the Stark shifts of the neutral and ionic ground states) is small at the intensities employed.

For 795 and $634 \mathrm{~nm}$, the photon energies are below the gap energy, and we observe that the $\mathrm{D}_{0}$ ATI progression is reproduced in the fragment ion PES (Fig. 2, lower panels). This observation shows that the dominant pathway for fragment ion formation under the conditions of this study is associated with direct $\mathrm{D}_{0}$ SFI followed by a sequential excitation processes in the 1-butene cation (pathway (ii)). SFI to the ionic ground state produces the $\mathrm{D}_{0}$ ATI comb. The ground-state ion, produced in a given field cycle, is further excited in subsequent laser cycles. As detailed in Appendix 1, we note that for photoelectron kinetic energies above $2 \mathrm{eV}$, the departing photoelectron never returns to less than 19 Bohr separation from the ion core after a propagation time of $2 \mathrm{fs}$ from its moment of birth. Thus, beyond possibly the first peak, the ATI progression is not expected to carry any information about the sequential excitation process.
Note that both for $\lambda=795 \mathrm{~nm}$ and $\lambda=634 \mathrm{~nm}$, the modulation depth for the fragment-correlated ATI spectra is significantly reduced as compared to the $\mathrm{D}_{0}$ ATI spectrum associated with the parent ion (see Fig. 1(a) and (b)). We suspect that the observation indicates a minor contribution of direct SFI to excited cation states in the fragment-associated PES for both wavelengths. We have confirmed that the observed reduction in modulation depth for the fragment channels, which are generally less intense than the parent channel, is not a measurement artefact of the covariance analysis. The covariant PES for the heavy 1-butene isotope ${ }^{13} \mathrm{C}^{12} \mathrm{C}_{3} \mathrm{H}_{8}{ }^{+}$, which constitutes $4.8 \%$ of the ion yield of ${ }^{12} \mathrm{C}_{4} \mathrm{H}_{8}{ }^{+}$(expected abundance $4.4 \%$ ), does not show a reduced modulation depth. Normalized PES of the two isotopes overlap very well, especially for lower kinetic energy, where the statistical error is lowest and the covariance analysis is expected to show the least errors.

For the case of $\lambda=795 \mathrm{~nm}$, we explicitly exclude the possibility that the alternative sequential pathway (iii) (neutral excitation, followed by ionization) plays a major role in 1-butene by performing an intensity-dependent measurement of the ATI spectra. Electronic excitation of neutral 1-butene involves population of a Rydberg orbital located at most $2.7 \mathrm{eV}$ below the ionization threshold. ${ }^{39}$ SFI of such Rydberg-like excited states preferentially removes the weakly-bound outer electrons, leaving the core intact. Because Stark shifts of Rydberg orbitals are typically very close to the free-electron ponderomotive shift, the ATI progressions formed by ionization of Rydberg-like neutral excited states are expected to be independent of the intensity. We observed, however, that both the parent- and fragment-associated ATI combs shift to lower photoelectron kinetic energies with increasing intensity (see Fig. 4(a) and (c)). This behaviour is inconsistent with a sequential pathway involving excitation to a neutral Rydberg state followed by ionization. Note that the shift observed in the parent-associated PES (Fig. 4(a)) precludes a sequential ionization pathway (iii) which produces a cation in the ionic ground state, while the shift observed in the fragmentassociated spectra precludes a sequential pathway (iii) which produces a cation in an electronically excited ionic state.

The intensity-dependent measurements of Fig. 4 also confirm that the differential Stark effects are small for the conditions employed in this study. According to eqn (1), the ATI comb shift contains both the intensity-dependent $U_{\mathrm{p}}$ and the differential Stark shift between the initial and final states, $\Delta E_{\text {Stark }}=\mathrm{IP}-\mathrm{IP}^{0}$, where the latter is the field-free value. In Fig. 4(b) and (d), we plot the absorbed photon energy $n \times h \nu$ minus the field-free vertical ionization potential $\mathrm{IP}^{0}=9.77 \mathrm{eV}^{42}\left(\mathrm{D}_{0}\right.$ ionization) minus the measured photoelectron kinetic energy $E_{\text {peak }}^{n}$ determined from the $n$th order ATI peaks shown in Fig. 4(a) and (c). In this manner, the values plotted in Fig. 4(b) and (d) for the intensities investigated here correspond to $U_{\mathrm{p}}+\Delta E_{\text {Stark }}$. The ponderomotive potential $U_{\mathrm{p}}$ is shown as the solid blue line. For the fragment and the parent ion data, a negative $\Delta E_{\text {Stark }}$ on the order of $0.2 \mathrm{eV}$ at $I=1.5 \times 10^{13} \mathrm{~W} \mathrm{~cm}^{-2}$ is found.

We note, however, that Fig. 4 reveals that the low kinetic energy range, up to $\approx 1.5 \mathrm{eV}$, displays a shape which changes with intensity and looks considerably different for parent- and 
fragment-associated spectra. This low energy region also features peaks which hardly shift with intensity (solid vertical lines), indicative of Freeman resonances. ${ }^{47}$ This observation concurs with the well-established fact that the low kinetic energy region of the ATI spectrum can be dominated by ionization processes which depend critically on the photon energy - in particular, resonance-enhanced multiphoton ionization ${ }^{33,34}$ - and thus may not correspond to (non-perturbative) SFI processes.

We now discuss the data obtained for SFI at $\lambda=401 \mathrm{~nm}$. As seen from the scheme in Fig. 5(c1), we expect the two lowest ATI orders for $\mathrm{D}_{0}$ SFI to appear at 2.5 and $5.6 \mathrm{eV}$ in the PES at this wavelength (with the differential Stark shifts again neglected, see above). Inspecting the ATI comb correlated with the ground-state cation, we find that this expectation agrees well with the dominant maxima located on the high kinetic energy side of the broad ATI peaks (see Fig. 1(c) and 2(c), upper panel). As is the case for $\lambda=795 \mathrm{~nm}$ and $\lambda=634 \mathrm{~nm}$ (see above), the $\mathrm{D}_{0}$ ATI progression is also reproduced in the fragment-associated ATI spectra (see comb marked in blue in Fig. 2(c), lower panel). This observation indicates that the sequential pathway (ii) is present and contributes strongly to the population of electronically excited cation states at $\lambda=401 \mathrm{~nm}$.

Importantly, however, in contrast to the case for photon energies below the gap energy, there is a very prominent second ATI progression appearing in the fragment-associated PES, marked in red in the lower panel of (Fig. 2(c)). This second ATI comb is shifted with respect to the $\mathrm{D}_{0}$ ATI progression to lower photoelectron energies by about $1.85 \mathrm{eV}$. A shift of this magnitude is close to the field-free gap energy of $2.0 \mathrm{eV}$. Given that small differential Stark shifts are expected and not accounted for, we assign the second ATI progression to $\mathrm{D}_{1} \mathrm{SFI}$, i.e. SFI producing directly the first excited cation state (see Fig. 5(c2)). The strong contribution of pathway (i) represents a marked difference for $\lambda=401 \mathrm{~nm}$ as compared to the two longer wavelengths.

The latter interpretation is supported by the comparison of fragment-associated PES acquired for pulse durations of $\tau=30 \mathrm{fs}$ and 110 fs at the same intensity, shown in Fig. 3. The $\mathrm{D}_{0}$ ATI comb is relatively stronger for the increased pulse duration. Indeed, we expect that the longer the SFI-created ground state $D_{0}$ cation is exposed to the strong laser field, the higher the probability will be that it will sequentially absorb additional photons that induce sequential fragmentation. Hence, when the laser pulse duration $\tau$ is increased at constant intensity, the relative yield of the sequential post-ionization excitation pathway increases. Consequently, a relatively more prominent $D_{0}$-like ATI comb is observed in the fragment-associated PES for longer pulse durations $\tau$.

As noted above, the peaks constituting the $\mathrm{D}_{0}$ ATI comb in the parent-associated PES are unusually broad for $\lambda=401 \mathrm{~nm}$. In particular, they are significantly broader than the peaks in the PES recorded for $\lambda=795 \mathrm{~nm}$ and $\lambda=634 \mathrm{~nm}$ (see Fig. 1). We have confirmed that this observation is not the result of the instrumental resolution of the REMI: the parent-associated PES for SFI of 1,3-butadiene, which was present as an impurity in small quantities in the gas target, displays much narrower ATI peaks at the expected energetic positions for $\lambda=401 \mathrm{~nm}$. Furthermore, located on the low kinetic energy side of the broad ATI peaks in the parent-associated PES from 1-butene, there are less pronounced maxima, which are shifted to lower kinetic energies by about $1 \mathrm{eV}$ compared to the main maxima assigned above (see Fig. 1(c)). These appear as prominent shoulders in the exponentially corrected spectra (see Fig. 2(c), upper panel). Two conclusions can be drawn: (1) Given that the ponderomotive energy is $0.1 \mathrm{eV}$ or less for all ionization events in the laser pulse, the $1 \mathrm{eV}$ photoelectron energy that is 'missing' compared to the $\mathrm{D}_{0}$ SFI ATI comb has to be tied up in internal molecular degrees of freedom. This excitation does not lead to molecular fragmentation on the timescale of the ion transit times of the extraction region of the mass spectrometer in our experiment, since the shoulders/secondary peaks appear predominantly in the parent-associated spectra. (2) Molecules excited in this way have a finite probability to be sequentially electronically excited, since the same features appear in the fragment-associated PES as well. However, since they are much weaker in the fragment spectra (see Fig. 1(c) and 2(c), lower panel), the sequential excitation probability is lower as compared to molecules produced by the dominant $\mathrm{D}_{0}$ SFI channel.

Via an NMR analysis we have excluded a significant contribution of the cyclobutane conformer, which possesses a vertical IP about $1 \mathrm{eV}$ higher than 1-butene, in the gas sample. Since the shoulders/secondary peaks observed in the $\mathrm{D}_{0}$-associated ATI progressions correspond to direct SFI which produces 1-butene cations that are internally excited by about $1 \mathrm{eV}$ (i.e. an energy below the $D_{0}-D_{1}$ gap) and appear prominently in the parentassociated spectrum, we speculate that SFI produces a contribution of vibrationally excited, stable parent cations. We note that, in principle, vibrational excitation slightly above the dissociation threshold can lead to hydrogen elimination on a timescale longer than the ion extraction region transit times in our experiment. Vertical single-photon ionization of 1-butene with VUV light results in a $D_{0}$ peak that is about $1 \mathrm{eV}$ wide and contains experimentally unresolved vibrational structure. ${ }^{36,37,48}$ The broad ATI peaks with a double-peak structure might involve a sequential process, where three-photon excitation leads to the formation of neutral excited states (Rydberg states) with a small binding energy. As soon as these states are excited, a rapid redistribution of electronic to vibrational energy will be initiated, possibly aided by internal conversion through conical intersections very close to the equilibrium geometry. ${ }^{49}$ To ionize, a fourth photon has to be absorbed within the laser pulse, limiting the extent of electronicto-vibrational energy transfer.

In order to independently corroborate the sequential excitation process, we have performed numerical time-dependent Schrödinger equation (TDSE) simulations of the survival probability (against excitation) of a ground-state 1-butene cation at the fixed neutral geometry. The cation was exposed to a $20 \mathrm{fs}$ (FWHM) duration laser pulse with a variable wavelength and a fixed Keldysh parameter. The orientationally averaged polarization was sampled on a 38-point order-9 Lebedev grid. Details of the calculation are described in Appendix 2. We emphasize that this model does not do justice to the full complexity of the problem and should be taken only as a qualitative indication of trends. The cation ground-state survival probability as a function 


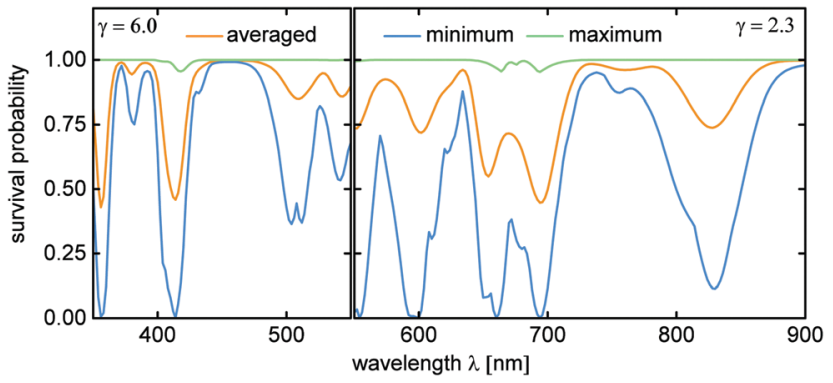

Fig. 6 Survival probability of a 1-butene ground-state cation as a function of wavelength, obtained from a TDSE simulation. In order to allow a comparison with the experimental results presented in this paper, the Keldysh parameter $\gamma$ was held constant at 2.3 and 6 for the two different wavelength regions, respectively. For a discussion, see the text.

of wavelength is plotted in Fig. 6 for $\gamma=2.3$ (wavelength range 550-900 nm) and $\gamma=6$ (wavelength range 350-550 nm). The orange curve represents the case where all molecular orientations with respect to the laser polarization are averaged. The green (blue) curve represents the maximum (minimum) survival probability found amongst these orientations. As can be seen, the probability that the ground-state cation is sequentially excited in the laser field is very significant over the entire studied wavelength range. These theory results therefore support the experimental finding that fragment formation via a sequential excitation process plays a prominent role, both for photon energies below and above the $\mathrm{D}_{0}-\mathrm{D}_{1}$ gap energy.

\section{Conclusions}

Our study of the strong-field ionization of the 1-butene molecule as a function of wavelength is complementary to previous work, where the wavelength was held constant but the target molecule was varied. ${ }^{31}$ There, it was observed that the relative fragment yield, corresponding to the sum of direct and indirect SFI to dissociative cation states, strongly decreases with increasing gap energy. Moreover, from analyzing coincidence photoelectron spectra, such a trend was confirmed individually for the two contributions. $^{31}$

In our study of 1-butene, we observed that the relative fragment yield decreases strongly with increasing SFI wavelength. We therefore find that the sum of direct and indirect SFI to dissociative cation states decreases strongly with decreasing photon energy, in qualitative agreement with the observation in the previous study. ${ }^{31}$ For photon energies below and near the gap energy, $\mathrm{D}_{0}$ SFI and sequential excitation is the dominant way of accessing $\mathrm{D}_{1}$, the dissociative lowest excited state of the 1-butene cation. A contribution from direct SFI to excited cation states may be the reason for a reduced peak contrast that is observed in the PES associated with fragment ion formation, but cannot be unambiguously identified. For a photon energy of about 1.5 times the gap energy, we observe a strong contribution of direct SFI to $\mathrm{D}_{1}$, along with the sequential excitation channel involving direct SFI to $D_{0}$ followed by further photo-absorption. The leading term of the ratio of the exponential Keldysh factors, ${ }^{35} \kappa=\exp \left[-2\left(2 \mathrm{IP}_{0}\right)^{1 / 2} \Delta \mathrm{IP} / F\right]$, used by the authors of the complementary previous work ${ }^{31}$ in the interpretation of their data, does not depend on the photon energy, as expected for a quasi-static theory. Its intensity dependence suggests a trend for the contribution of excited state SFI opposite to that we experimentally observed: $\kappa$ decreases from $9 \times 10^{-3}$ for $I=2.5 \times 10^{13} \mathrm{~W} \mathrm{~cm}^{-2}(634 \mathrm{~nm})$ to $2 \times 10^{-3}$ for $I=1.5 \times$ $10^{13} \mathrm{~W} \mathrm{~cm}^{-2}(795 \mathrm{~nm})$ and to $6 \times 10^{-4}$ for $I=1.0 \times 10^{13} \mathrm{~W} \mathrm{~cm}^{-2}$ (401 nm). In contrast, we observed a strongly increased contribution of excited state SFI for the low-intensity $401 \mathrm{~nm}$ wavelength measurement, in qualitative disagreement with the Keldysh rate predictions.

While we can clearly identify the mechanism behind the cation excited state population, further work is needed to determine the nature of the transition from sequential to direct excitation as the photon energy increases. We found evidence for vibrational excitation during SFI at the highest photon energy and speculate that nuclear (vibrational) dynamics might play a role in a potential sequential ionization pathway. We remark that nuclear dynamics was very recently confirmed to play a role in post-ionization excitation. ${ }^{50,51}$ Future studies of the correlated photoelectron spectra as a function of intensity, pulse duration, and wavelength within a pump-probe scheme would be very helpful for developing a deeper understanding, in particular for photon energies above the gap energy. Finally, it would be interesting to explore how general our conclusions are by performing similar studies in other molecular systems.

\section{Conflicts of interest}

There are no conflicts to declare.

\section{Appendix 1: departure of the photoelectrons from the ion core}

Neglecting the magnetic component of the laser field and the molecular potential, motion of a classical electron in a strong laser field is a combination of the uniform drift and the fielddriven oscillation (atomic units: $\hbar=m=-e=1$ ):

$$
x=v_{\mathrm{d}} t+L_{0} \cos \left(\omega t+\phi_{0}\right)-L_{0} \cos \left(\phi_{0}\right),
$$

where we assume that the electron enters the field at time zero at the coordinate origin. The drift velocity $v_{\mathrm{d}}=\sqrt{2 E}$ is determined by the kinetic energy $E$ recorded at the detector. The oscillation radius $L_{0}$ depends on the laser field parameters via $L_{0}=F_{0} / \omega^{2}$, where $F_{0}$ is the peak electric field and $\omega$ is the laser frequency. Finally, $\phi_{0}$ is the phase of the laser electric field at time zero. Regardless of the time and the initial field phase, the classical trajectory will always remain within $2 L_{0}$ of the drift displacement $v_{\mathrm{d}} t$. For the field parameters in this study (see Table 1), the free-oscillation radius is 6.3, 5.2, and $1.4 \mathrm{Bohr}$, respectively for the 795, 635, and $401 \mathrm{~nm}$ driving fields. A photoelectron with $E=2 \mathrm{eV}$ energy at the detector $\left(v_{\mathrm{d}} \approx 0.4\right.$ atomic units) will drift by 32 Bohr within the first $2 \mathrm{fs}$ ( $\approx 83$ atomic units), and will not return to less than 19 Bohr from the origin for any field considered here. This separation is more than twice the extent of 
Table 2 MR-CIS states used for modeling of sequential excitation of the ground-state cation

\begin{tabular}{|c|c|c|c|c|c|c|}
\hline State & $E_{\text {tot }}[$ Hartree $]$ & $E_{\text {rel }}[\mathrm{eV}]$ & $\langle$ dys $|$ dys $\rangle$ & $E_{\text {rel }}[\mathrm{MCQDPT} 2]$ & Lead det. & Character \\
\hline 1 & -155.93707 & 0.000 & 0.8897 & 0.000 & $(\mathrm{H}):$ & (pi) \\
\hline 2 & -155.87000 & 1.825 & 0.9013 & 2.141 & $(\mathrm{H}-1):$ & $(\mathrm{C} 2-\mathrm{C} 3)$ \\
\hline 3 & -155.85228 & 2.307 & 0.9109 & 2.465 & $(\mathrm{H}-2):$ & $(\mathrm{C} 3-\mathrm{H})$ \\
\hline 4 & -155.82706 & 2.994 & 0.8951 & 3.188 & $(\mathrm{H}-3):$ & $(\mathrm{C} 3-\mathrm{C} 4)$ \\
\hline 5 & -155.80923 & 3.479 & 0.9016 & 3.553 & $(\mathrm{H}-4):$ & $(\mathrm{C} 1-\mathrm{C} 2, \mathrm{C} 1-\mathrm{H}, \mathrm{C} 2-\mathrm{H}, \mathrm{C} 4-\mathrm{H})$ \\
\hline 6 & -155.76401 & 4.709 & 0.8798 & 4.757 & $(\mathrm{H}-5):$ & $(\mathrm{C} 1-\mathrm{H}, \mathrm{C} 4-\mathrm{H})$ \\
\hline 7 & -155.74826 & 5.138 & 0.8751 & $\mathrm{n} / \mathrm{c}$ & $(\mathrm{H}-6):$ & $(\mathrm{C} 1-\mathrm{H}, \mathrm{C} 4-\mathrm{H})$ \\
\hline 8 & -155.71521 & 6.037 & 0.8365 & $\mathrm{n} / \mathrm{c}$ & $(\mathrm{H}-7):$ & $(\mathrm{C} 1-\mathrm{H}, \mathrm{C} 3-\mathrm{H})$ \\
\hline 9 & -155.69561 & 6.570 & 0.0205 & $\mathrm{n} / \mathrm{c}$ & $(\mathrm{H})(\mathrm{H}) \mathrm{L}:$ & Norm from (C1[p]) \\
\hline 10 & -155.67432 & 7.150 & 0.0316 & $\mathrm{n} / \mathrm{c}$ & $(\mathrm{H})(\mathrm{H}-1) \mathrm{L}:$ & \\
\hline 11 & -155.66693 & 7.351 & 0.0052 & $\mathrm{n} / \mathrm{c}$ & $(\mathrm{H})(\mathrm{H}-2) \mathrm{L}:$ & \\
\hline 12 & -155.62657 & 8.449 & 0.3023 & $\mathrm{n} / \mathrm{c}$ & $(\mathrm{H}-8)+(\mathrm{H})(\mathrm{H}-3) \mathrm{L}+1$ & \\
\hline 13 & -155.62107 & 8.599 & 0.2779 & $\mathrm{n} / \mathrm{c}$ & $(\mathrm{H}-8)+(\mathrm{H})(\mathrm{H}-3) \mathrm{L}+1$ & \\
\hline 14 & -155.60654 & 8.994 & 0.2161 & $\mathrm{n} / \mathrm{c}$ & $(\mathrm{H}-8)+(\mathrm{H})(\mathrm{H}-3) \mathrm{L}+1$ & \\
\hline 15 & -155.56177 & 10.212 & 0.0044 & $\mathrm{n} / \mathrm{c}$ & $(\mathrm{H})(\mathrm{H}-5) \mathrm{L}+1$ & \\
\hline 16 & -155.55292 & 10.453 & 0.0985 & $\mathrm{n} / \mathrm{c}$ & $(\mathrm{H})(\mathrm{H}-6) \mathrm{L}+1$ & \\
\hline 17 & -155.54955 & 10.545 & 0.0376 & $\mathrm{n} / \mathrm{c}$ & Very mixed & \\
\hline 18 & -155.54775 & 10.594 & 0.0287 & $\mathrm{n} / \mathrm{c}$ & $(\mathrm{H}-1)(\mathrm{H}-1) \mathrm{L}+1$ & \\
\hline 19 & -155.53612 & 10.910 & 0.5747 & $\mathrm{n} / \mathrm{c}$ & $(\mathrm{H}-9)$ & \\
\hline 20 & -155.53298 & 10.996 & 0.0003 & $\mathrm{n} / \mathrm{c}$ & $(\mathrm{H}-1)(\mathrm{H}-2) \mathrm{L}+1$ & \\
\hline 21 & -155.52940 & 11.093 & 0.0531 & $\mathrm{n} / \mathrm{c}$ & $(\mathrm{H})(\mathrm{H}-2) \mathrm{L}+1$ & \\
\hline
\end{tabular}

the long molecular axis of the 1-butene molecule (the $\mathrm{C}_{1}-\mathrm{C}_{4}$ distance is about 6.8 atomic units ${ }^{52}$ ). At this distance, changes in the molecular potential due to sequential, one-photon excitation of the cation are expected to decrease below our experimental resolution $(\approx 0.2 \mathrm{eV})$. This estimation will be necessarily modified by the quantum spreading of the photoelectron wave packet and the effects of the long-range binding potential. Nonetheless, we do not expect higher-order ATI peaks to carry any information on the sequential excitation processes in the cation core.

\section{Appendix 2: methods for theoretical modeling of cation excitation}

All electronic structure calculations utilized a aug-cc-pVTZ basis set. The nuclear geometry of the staggered neutral conformer was optimized at the MP2(fc) level. The cationic excited state energies and transition dipoles at this geometry were determined in a Multi-Reference Configuration Interaction with Single excitations (MR-CIS) calculation. The reference orbitals were determined in a $\operatorname{CASSCF}(23,13)$ calculation. The CASSCF active space contained all valence occupied orbitals, plus the $\pi^{*}$ virtual orbital, and was optimized for the eight, equallyweighted lowest states of the cation. In the MR-CIS calculation, excitations to all virtual orbitals below +2 Hartree were allowed (193 external orbitals), with the full $\operatorname{CAS}(23,13)$ used as the reference space. The lowest 21 doublet MR-CIS states detailed in Table 2 were used as the basis for the TDSE calculation. For the lowest six states, we confirmed that the MR-CIS energies are in a good agreement with correlated ORMAS(15,12,SDT)-PT2/ aug-cc-pVDZ/aug-cc-pVDZ ["MCQDPT2"] results.

TDSE calculations in the basis of MR-CIS states used a truncated Gaussian pulse (20 fs FWHM). The peak intensity was chosen to maintain constant Keldysh parameter $\gamma=2.3$ (6.0) for each wavelength. The ground state of the cation was assumed to be stable. All excited states were assigned a natural decay width of $\Gamma=1 \mathrm{meV}$. The TDSE was integrated numerically to 8-digit accuracy, using the NDSolveValue built-in function in Mathematica.

\section{Acknowledgements}

We would like to acknowledge Doug Moffat and Malgosia Daroszewska (both NRC) and Katrin Herrmann (MBI) and Sebastian Kemper (TU Berlin) for their NMR analysis of the gas samples. We also thank Roman Peslin from the A2 mechanical workshop at MBI for excellent support. The work at the MBI has been supported by the German Science Foundation (DFG SCHU 645/8-1). AS acknowledges the NSERC Discovery grant program for financial support.

\section{Notes and references}

1 F. Krausz and M. Ivanov, Rev. Mod. Phys., 2009, 81, 163.

2 M. Y. Ivanov, M. Spanner and O. Smirnova, J. Mod. Opt., 2005, 52, 165.

3 P. B. Corkum, Phys. Rev. Lett., 1993, 71, 1994.

4 J. L. Krause, K. J. Schafer and K. C. Kulander, Phys. Rev. Lett., 1992, 68, 3535.

5 A. N. Pfeiffer, C. Cirelli, M. Smolarski and U. Keller, Chem. Phys., 2013, 414, 84.

6 S. Baker, J. S. Robinson, C. A. Haworth, H. Teng, R. A. Smith, C. C. Chirila, M. Lein, J. W. G. Tisch and J. P. Marangos, Science, 2006, 312, 424.

7 W. Li, X. Zhou, R. Lock, S. Patchkovskii, A. Stolow, H. C. Kapteyn and M. M. Murnane, Science, 2008, 322, 1207. 8 M. Spanner, J. Mikosch, A. E. Boguslavskiy, M. M. Murnane, A. Stolow and S. Patchkovskii, Phys. Rev. A: At., Mol., Opt. Phys., 2012, 85, 033426.

9 M. Meckel, D. Comtois, D. Zeidler, A. Staudte, D. Pavicic, H. C. Bandulet, H. Pepin, J. C. Kieffer, R. Doerner, D. M. Villeneuve and P. B. Corkum, Science, 2008, 320, 1478. 
10 P. Agostini, F. Fabre, G. Mainfray, G. Petite and N. Rahman, Phys. Rev. Lett., 1979, 42, 1127.

11 M. Spanner, J. Mikosch, A. Gijsbertsen, A. E. Boguslavskiy and A. Stolow, New J. Phys., 2011, 13, 093010.

12 M. Lezius, V. Blanchet, D. M. Rayner, D. M. Villeneuve, A. Stolow and M. Y. Ivanov, Phys. Rev. Lett., 2001, 86, 51.

13 A. E. Boguslavskiy, J. Mikosch, A. Gijsbertsen, M. Spanner, S. Patchkovskii, N. Gador, M. J. J. Vrakking and A. Stolow, Science, 2012, 335, 1336.

14 M. Kotur, C. Zhou, S. Matsika, S. Patchkovskii, M. Spanner and T. C. Weinacht, Phys. Rev. Lett., 2012, 109, 203007.

15 J. Mikosch, A. E. Boguslavskiy, I. Wilkinson, M. Spanner, S. Patchkovskii and A. Stolow, Phys. Rev. Lett., 2013, 110, 023004 .

16 V. Tagliamonti, H. Chen and G. N. Gibson, Phys. Rev. Lett., 2013, 110, 073002.

17 O. Njoya, S. Matsika and T. Weinacht, Chem. Phys. Chem., 2013, 14, 1451.

18 F. Calegari, D. Ayuso, A. Trabattoni, L. Belshaw, S. De Camillis, S. Anumula, F. Frassetto, L. Poletto, A. Palacios, P. Decleva, J. B. Greenwood, F. Martin and M. Nisoli, Science, 2014, 346, 336.

19 O. Smirnova, Y. Mairesse, S. Patchkovskii, N. Dudovich, D. M. Villeneuve, P. B. Corkum and M. Y. Ivanov, Nature, 2009, 460, 972.

20 B. K. McFarland, J. P. Farrell, P. H. Bucksbaum and M. Gühr, Science, 2008, 322, 1232.

21 H. Akagi, T. Otobe, A. Staudte, A. Shiner, F. Turner, R. Doerner, D. M. Villeneuve and P. B. Corkum, Science, 2009, 325, 1364.

22 M. Oppermann, S. J. Weber, L. J. Frasinski, M. Y. Ivanov and J. P. Marangos, Phys. Rev. A: At., Mol., Opt. Phys., 2013, 88, 043432.

23 Q. Y. Ji, S. Cui, X. Y. You, X. C. Gong, Q. Y. Song, K. Lin, H. F. Pan, J. X. Ding, H. P. Zeng, F. He and J. Wu, Phys. Rev. A: At., Mol., Opt. Phys., 2015, 92, 043401.

24 M. Uiberacker, T. Uphues, M. Schultze, A. J. Verhoef, V. Yakovlev, M. F. Kling, J. Rauschenberger, N. M. Kabachnik, H. Schroeder, M. Lezius, K. L. Kompa, H.-G. Muller, M. J. J. Vrakking, S. Hendel, U. Kleineberg, U. Heinzmann, M. Drescher and F. Krausz, Nature, 2007, 446, 627.

25 E. Goulielmakis, Z.-H. Loh, A. Wirth, R. Santra, N. Rohringer, V. S. Yakovlev, S. Zherebtsov, T. Pfeifer, A. M. Azzeer, M. F. Kling, S. R. Leone and F. Krausz, Nature, 2010, 466, 739.

26 Z.-H. Loh and S. R. Leone, J. Phys. Chem. Lett., 2013, 4, 292. 27 J. Mikosch and S. Patchkovskii, J. Mod. Opt., 2013, 60, 1426. 28 J. Mikosch and S. Patchkovskii, J. Mod. Opt., 2013, 60, 1439.

29 L. J. Frasinski, K. Codling and P. A. Hatherly, Science, 1989, 246, 1029.

30 P. Sandor, A. Zhao, T. Rozgonyi and T. C. Weinacht, J. Phys. B: At., Mol. Opt. Phys., 2014, 47, 124021.

31 A. Zhao, P. Sandor, T. Rozgonyi and T. C. Weinacht, J. Phys. B: At., Mol. Opt. Phys., 2014, 47, 204023.
32 W. D. M. Lunden, D. Geißler, P. Sandor, T. C. Weinacht and T. Rozgonyi, Phys. Rev. A: At., Mol., Opt. Phys., 2014, 89, 053404.

33 P. Sandor, V. Tagliamonti, A. Zhao, T. Rozgonyi, M. Ruckenbauer, P. Marquetand and T. Weinacht, Phys. Rev. Lett., 2016, 116, 063002.

34 V. Tagliamonti, P. Sandor, A. Zhao, T. Rozgonyi, P. Marquetand and T. Weinacht, Phys. Rev. A: At., Mol., Opt. Phys., 2016, 93, 051401.

35 L. V. Keldysh, Zh. Eksp. Teor. Fiz., 1964, 47, 1945.

36 F. Wu, X. J. Chen, X. Shan, S. X. Tian, Z. J. Li and K. Z. Xu, J. Phys. Chem. A, 2008, 112, 4360.

37 R. M. White, T. A. Carlson and D. P. Spears, J. Electron Spectrosc. Relat. Phenom., 1974, 3, 59.

38 M. Kunitski, S. Knippenberg, A. Dreuw and B. Brutschy, Vib. Spectrosc., 2011, 56, 13.

39 E. Es-sebbar, Y. Benilan and A. Farooq, J. Quant. Spectrosc. Radiat. Transfer, 2013, 115, 1.

40 J. Mikosch, C. Z. Bisgaard, A. E. Boguslavskiy, I. Wilkinson and A. Stolow, J. Chem. Phys., 2013, 139, 024304.

41 Z. Sacks, G. Mourou and R. Danielius, Opt. Lett., 2001, 26, 462 .

42 D. A. Krause, J. W. Taylor and R. F. Fenske, J. Am. Chem. Soc., 1978, 100, 718.

43 J. Ullrich, R. Moshammer, A. Dorn, R. Dörner, L. P. H. Schmidt and H. Schmidt-Böcking, Rep. Prog. Phys., 2003, 66, 1463.

44 Venteon vCHIRP Software, Laser Quantum.

45 A. Ferre, A. E. Boguslavskiy, M. Dagan, V. Blanchet, B. D. Bruner, F. Burgy, A. Camper, D. Descamps, B. Fabre, N. Fedorov, J. Gaudin, G. Geoffroy, J. Mikosch, S. Patchkovskii, S. Petit, T. Ruchon, H. Soifer, D. Staedter, I. Wilkinson, A. Stolow, N. Dudovich and Y. Mairesse, Nat. Commun., 2015, 6, 5952.

46 C. E. Van der Meij, J. van Eck and A. Niehaus, Chem. Phys., 1989, 130, 325.

47 R. R. Freeman, P. H. Bucksbaum, H. Milchberg, S. Darack, D. Schumacher and M. E. Geusic, Phys. Rev. Lett., 1987, 59, 1092.

48 G. Bieri, F. Burger, E. Heilbronner and J. Maier, Helv. Chim. Acta, 1977, 60, 2213.

49 M. C. E. Galbraith, S. Scheit, N. V. Golubev, G. Reitsma, N. Zhavoronkov, N. Despré, F. Lépine, A. I. Kuleff, M. J. J. Vrakking, O. Kornilov, H. Köppel and J. Mikosch, Nat. Commun., 2017, 8, 1018.

50 A. Zhao, P. Sandor, V. Tagliamonti, T. Rozgonyi, P. Marquetand and T. Weinacht, Phys. Rev. A: At., Mol., Opt. Phys., 2017, 96, 023404.

51 V. Tagliamonti, B. Kaufman, A. Zhao, T. Rozgonyi, P. Marquetand and T. Weinacht, Phys. Rev. A: At., Mol., Opt. Phys., 2017, 96, 021401(R).

52 K. H. Hellwege and A. M. E. Hellwege, Landolt-Bornstein: Group II: Atomic and Molecular Physics Volume 7: Structure Data of Free Polyatomic Molecules, Springer, 1976. 